\title{
The 'Holy Grail' in Immuno-Oncology: AC BioScience SA is Aiming to Potentiate Anti-PD-1 Therapy Efficacy through Tumor Cell Conditioning Strategy
}

\author{
Andreas Schläpfer ${ }^{\star a}$, Christian Auclair ${ }^{a}$, Bassam Janjib, Manale Karamª, and Muhammad Zaeem \\ Noman ${ }^{\mathrm{b}}$
}

\begin{abstract}
AC BioScience is a Swiss biotech company based at the EPFL Innovation Park and Biopôle, dedicated to developing groundbreaking therapies to fight a range of cancers and infectious diseases. We are about to start clinical trials with two of four leading-edge cancer drugs mainly focusing on immune-oncology and tumor vascular normalization with multi-billion \$ sales potential. Here, we present our strategy and one of our pioneering drug candidates that has already shown exceptional results with tumor cell conditioning to improve the efficacy of immune checkpoint inhibitors.
\end{abstract}

Keywords: AC Bioscience SA · ACB1801 · Cancer therapies · Immune checkpoint inhibitors · Swiss biotech

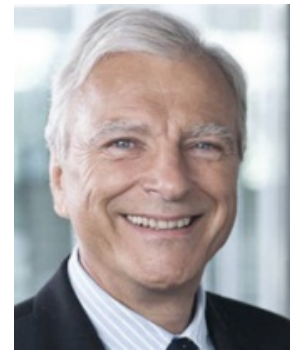

Nutrition business.

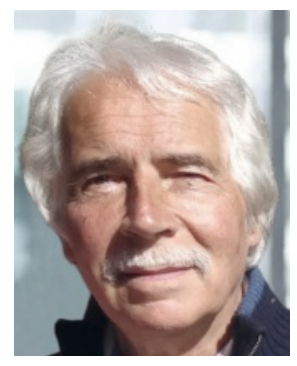

Chief Executive Officer and co-founder is Andreas Schläpfer, who has extensive experience in business strategy and global management. He holds a Master's Degree in Economics from St. Gallen University. He spent more than three decades as a senior executive at the Nestlé Group (CEO in France, Russia, Thailand/Indochina) and as Global Business Head/Senior Vice President in charge of Nestlé's Infant Head of the Department of Biochemistry Bioengineering at the Ecole Normale Supérieure de Cachan He co-founded the Graduate School of Oncology at the Institut Gustave Roussy and founded the Master's programme in Cancer Research.

\section{Business Case, Context, Motivation}

During his life-long research activities and senior role in the leading cancer research organizations in France, Professor Christian Auclair gained a unique insight and translational knowledge of oncology-related activities carried out over the years. He also noted that a number of discoveries in basic research had not been pursued further because of shifting research priorities and altered budget allocations. Some of the molecules at the center of this research held exceptional promise for the lives and survival rates of innumerable cancer patients.

When Professor Auclair met Andreas Schläpfer, a former senior executive at the Nestlé Group, the two came to the decision that the most promising of these molecules should be taken through (pre)clinical research, with a vision to aid cancer patients in need. As a result, AC BioScience SA was created at the EPFL Innovation Park near Lausanne in March 2017.

\section{Solution, Scientific Approach}

The huge progress in cancer research in the past two decades has led to a renewed interest in combining conventional treatments based on new rationales. The realization that cancer progression is not exclusively due to cancer deregulated cell proliferation, but also involves the nature of tumor microenvironment and the global immune response has opened new ways for the design of new combination treatments.

AC BioScience is pioneering the development of several ground breaking cancer therapies, two of which will start with clinical trials in 2021: One of its innovative approaches aims to improve the treatment of metastatic pancreatic adenocarcinoma using a proangiogenic S1P lyase inhibitor acting as a tumor vascular normalizer that will help drugs to better access solid tumors and oxygenation. A successful proof of concept would provide a premise for a similar strategy to be implemented for all poorly vascularized solid tumors, thus, providing a transformative new standard for cancer treatment.

AC BioScience's second drug candidate for clinical trial is a beta-carboline derivative in a combination treatment. It deals with tumor cell conditioning to render cancer therapy more responsive to cancer treatment resulting from either genetic or pharmacologic manipulation of the malignant phenotype. Here AC BioScience presents an example of tumor cell conditioning to improve the efficacy of immune checkpoint inhibitors. 


\subsection{Breakthrough in Cancer Treatment: Immune Checkpoint Inhibitors}

During the past few years, immune checkpoints that maintain physiologic self-tolerance have been shown to be involved in the downregulation of anti-tumor immunity. As typical example, cytotoxic T-lymphocyte antigen 4 (CTLA-4) plays an important role in the regulation of immune activation and tolerance; CTLA-4 signaling inhibits T-cell activation, particularly during strong T-cell responses. In the same line, the binding of Programmed Cell Death Protein 1 (PD-1), a receptor expressed on activated $\mathrm{T}$ cells, to its ligands, PD-L1 and PD-L2, negatively regulates immune responses. The PD-1 ligands are found on most cancers, and PD-1/PD-L1/2 interaction inhibits $\mathrm{T}$ cell activity and allows cancer cells to escape immune surveillance.

The strategies to restore anti-tumor immunity have focused at first on monoclonal antibody-based targeting of CTLA-4 and afterward PD-1 on T lymphocytes and its main ligand (PD-L1) on tumor cells. Food and Drug Administration (FDA) has approved various anti-PD-1/PD-L1 monoclonal antibodies (mAbs) for the treatment of metastatic melanoma, renal cell carcinoma (RCC), non-small cell lung cancer (NSCLC), bladder cancer, recurrent or metastatic head and neck cancer, refractory classical Hodgkin lymphoma, colorectal cancer (CRC), and metastatic Merkel cell carcinoma. Despite these successes, many patients experience intrinsic resistance, and even responding patients can develop acquired resistance to anti-PD-1 therapy.[1] In patients suffering from metastatic melanoma, the objective response rates remain limited and are around $20 \%$ to $25 \%$ for the patients treated with anti-CTLA-4[2] and roughly $30-35 \%$ for PD-L1 positive patients treated with anti-PD-1/PD-L1.[3]

Numerous studies are currently going on to elucidate the mechanisms responsible for $\mathrm{mAbs}$ resistance and exploring effective combinatorial approaches to recover efficacy or increase sensitivity in PD-1 blockade-resistant patients is attracting research attention.

\subsection{Tumor Antigen Presentation as Key of Immune Response}

The cancer-immunity mechanism indicates that CD8 $\mathrm{T}$ cell recognition of neoantigens (arising from altered tumor proteins formed as a result of tumor mutations) displayed by the major histocompatibility complex class I (MHC-I) on tumor cells is a critical step to eliminate these tumorigenic cells via immune checkpoint inhibitors therapy. ${ }^{[4]}$ In this context, it has been observed that the tumor mutation burden (TMB), was a predictive marker of checkpoint blockade therapy efficacy. ${ }^{[5]}$ However, the CD8 T cell cytotoxic response depends on the efficacy of the MHC-I mediated antigen presentation machinery which is a multistep complex process often impaired in tumor cells.

Tumor antigens are first processed to peptides by the immunoproteasome. Then, these antigenic peptides are translocated from the cytosol into the endoplasmic reticulum (ER) by the transporter associated with antigen processing (TAP) which also acts as a scaffold for the final stage of MHC class I assembly. ${ }^{[6]}$ In the ER, MHC-I molecules are formed by the folding of the heavy chain $\alpha$-subunit and its assembly with the $\beta 2 \mathrm{~m}$ subunit with the assistance of ER resident chaperons. Then, the peptide-loading complex (PLC) is formed by association of MHC-I molecule, calreticulin and ERP57 chaperons, tapasin and TAP. ${ }^{[7]}$ Tapasin acts as a bridging molecule between the MHC class I/chaperone complex and TAP facilitating the binding of high affinity peptides to the MHC-I molecules. After peptide loading, MHC-I molecules dissociate from PLC to export sites on the ER membrane where they are selectively recruited into cargo vesicles for transport to the Golgi apparatus. ${ }^{[8]}$ MHC-I/peptide complex then traffics through the Golgi apparatus to the plasma membrane (Fig. 1)

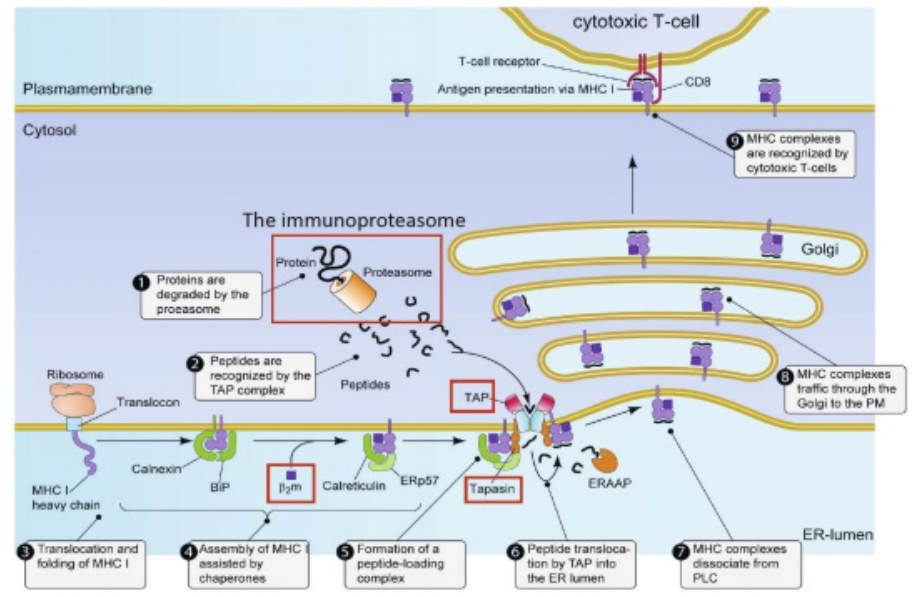

Fig. 1. Schematic representation of MHC-I-mediated antigen processing and presenting machinery. BiP, immunoglobulin binding protein; $\beta 2 \mathrm{~m}$, $\beta 2$-microglobulin; ER, endoplasmic reticulum; ERAP, ER-resident aminopeptidase (trims peptides to 8-10 mers); MHC I, major histocompatibility complex class I; PLC, peptide-loading complex; TAP, transporter associated with antigen processing (adapted from ref. [9]: Abele and Tampe, 2004).

\subsection{Impairment of MHC-I-mediated Antigen Presentation in Tumor Cells}

As mentioned above, the MHC-I antigen presentation machinery is often impaired in tumor cells and it is well established that tumor immune escape is associated with MHC-I downregulation, as seen in different human and experimental tumors and reviewed in many reports. ${ }^{[10]}$ MHC-I expression depends on the tumor phenotype and two extreme phenotypes can be identified: a good phenotype (rejected tumor phenotype) and a bad phenotype (escape tumor phenotype) (Fig. 2). Tumors with the later profile can be derived from tumors that got established and progressed after they had escaped T-cell mediated immunosurveillance. [10a] A tumor derived from an HLA-I positive epithelium can lose totally or partially the expression of class I molecules. ${ }^{[10 a]}$ The total percentage of various types of HLA-I loss, including total loss, haplotype loss, or allelic loss, ranges from 65 to $90 \%$, depending on the type of cancer. ${ }^{[11]}$
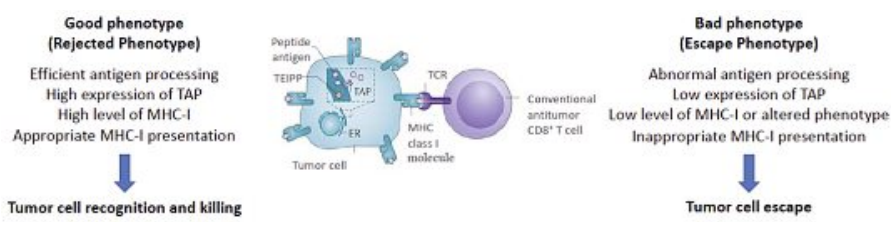

Fig. 2. Tumor phenotype heterogeneity conditions the tumor escape from immune surveillance. ER, endoplasmic reticulum; MHC-I, major histocompatibility complex class I; TAP, transporter associated with antigen processing; TCR, T-cell receptor; TEIPP, T cell epitopes associated with impaired peptide processing.

The relevance of the phenotypic classification is provided as a typical example by colorectal cancer (CRC) patients in which $35 \%$ of them have an abnormal MHC-I expression. It has been observed in CRC that TAP1 down-regulation elicits immune escape and poor prognosis. ${ }^{[12]}$ This observation can be extended to most tumor types as assessed by a recent elegant work done by Wang et al. ${ }^{[13]}$ In this study, the authors proposed a method to measure tumor immunogenicity score (TIGS), which combines tumor mutational burden (TMB) and an expression signature of the antigen processing and presenting machinery (APM). A clear correlation exists between pan-cancer immune checkpoint inhibitors objective response rates (ORR) and immune tumor status for 


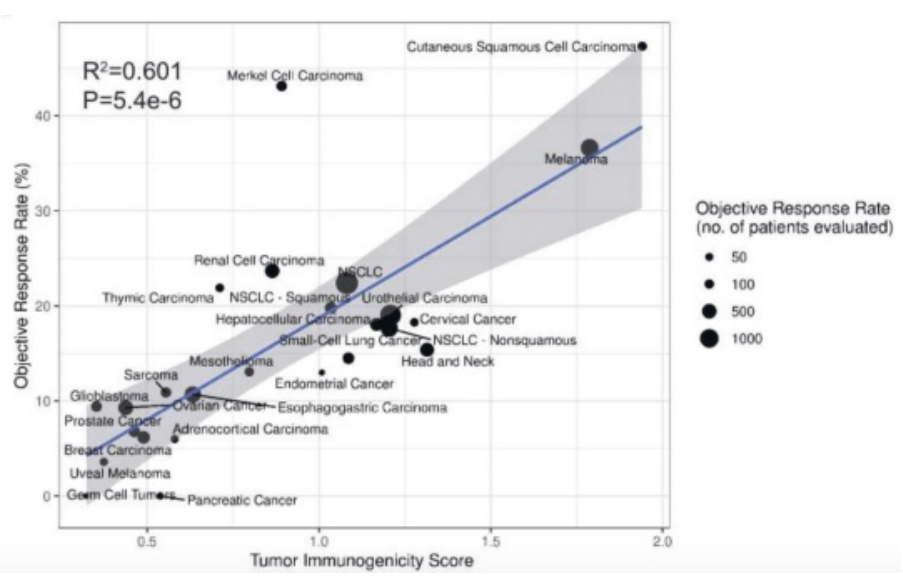

Fig. 3. Tumor immunogenicity score and predicted pan-cancer response rates to PD-1 inhibition. Correlation between Tumor immunogenicity score (TIGS) and objective response rate (ORR) with anti-PD-1 as described in published studies for which data regarding the ORR is available. The number of patients who were evaluated for the ORR is shown for each tumor type (size of the circle) (from ref. [13], Wang et al., 2019).

individual patients (Fig. 3). Data in Fig. 3 clearly shows that TIGS is an effective tumor-inherent biomarker for response prediction.

It is obvious that we have a limited possibility to increase the intrinsic tumor mutational burden and the TIGS increase should be obtained by improving the antigen presentation machinery efficacy. Along this line, the challenge facing the researchers is how to turn tumor cells escape phenotype into antigen presenting cell phenotype (rejected phenotype) (Fig. 2).

\subsection{The Tumor Phenotype Reversion: The Strategy to Improve Tumor Antigen Presentation}

Among the suspected mechanisms of resistance to immune checkpoint targeting, AC BioScience has focused investigations on the expression of MHC-I by tumor cells and on the relationship existing between the malignant phenotype and the MHC-I functional integrity.

As with many parasites, to survive and proliferate, tumor cells should escape from the immune surveillance of the host. The host immune response can be considered as a selection pressure and according to the evolution theory, the genetic program behind the malignant phenotype provides an adaptive response allowing the escape from the immune system. The impairment of antigen presentation is one of the consequences of this adaptive response.

In this context, it is expected that the 'manipulation' of the malignant phenotype; i.e. the loss of all or part of the malignant characters (referred to as tumor reversion), would result in the unmasking of tumor cells mainly through the rescue of tumor antigen presentation.

Along this line, several years ago a scientific program started at Gustave Roussy Institute (Villejuif France) aiming to identify the specific molecular basis of malignant phenotype maintenance and key processes of which pharmacological manipulation would result in tumor phenotypic reversion. In agreement with previous observations, it was hypothesized that changes in the cytoskeletal architecture which is one of the main molecular mechanisms underlying tumor progression could be a pertinent target process for the induction of tumor phenotype reversion. Consistently, genetic-induction of actin network rearrangement in transformed cells resulted in phenotypic reversion thanks to the rescue of cell adhesion and motility controls. ${ }^{[14]}$ Based on these findings, a two-step screening strategy involving a molecular primary screening (actin dynamics modulation) followed by a cellular functional secondary screening (cell adhesion and motility) have been performed. Several compounds in the series of pyridoindole and pyridocarbazole were found to promote actin cytoskeleton remodelling and act as tumorreverting agent at non-cytotoxic concentration. ${ }^{[15]}$ One of these compounds (ACB1801) has been selected by AC BioScience to start, in close collaboration with the Luxembourg Institute of Health (LIH), a series of proof of concept studies using murine melanoma B16-F10, a tumor experimental model extremely resistant to anti PD-1 antibody.

\section{Product/Service and Outlook: ACB1801 Elicits the MHC-I Machinery and Potentiates Anti-PD-1 in Melanoma B16-F10 Model}

The murine B16-F10 melanoma model is the most used metastatic melanoma model for preclinical studies. The syngeneic B16-F10 model allows the evaluation of responses to immuno-oncology agents in immunocompetent C57BL/6 mice. In this model, checkpoint inhibitors anti-mouse PD-1 or anti-mouse PD-L1 did not produce any response in subcutaneous B16-F10 tumors. Similarly, initiation of treatment with anti-mouse CTLA-4 or antimouse LAG-3 as early as four days post implant did not produce any response as well. Thus, melanoma B16-F10 is an experimental tumor model resistant to checkpoint inhibitors and therefore appropriate to investigate a strategy dealing to the transformation of a resistant phenotype to responder phenotype.

In close collaboration with the LIH team headed by Dr. Bassam Janji, we have extensively investigated the effect of ACB1801 on the different components of the antigen presentation machinery, the MHC-I antigen presentation and the consequences in terms of anti-PD-1 antitumor efficacy. Some of the key results are presented and discussed below.

\subsection{ACB1801 Strongly Increases MHC-I-mediated Antigen Presentation in Melanoma B16-F10 Cells}

Melanoma B16-F10 treatment with ACB1801 results in a dose-dependent increase in $\mathrm{H}-2 \mathrm{~Kb}$ bound OVA (SIINFEKL) peptide presentation as promoted by MHC-I complex at the cell membrane. Starting from $25 \mu \mathrm{M}$ ACB1801, the increasing effect is close to the one induced by interferon gamma (Fig. 4). It should be noted that up to $50 \mu \mathrm{M}, \mathrm{ACB} 1801$ has no effect on the melanoma cell viability and cell cycle.

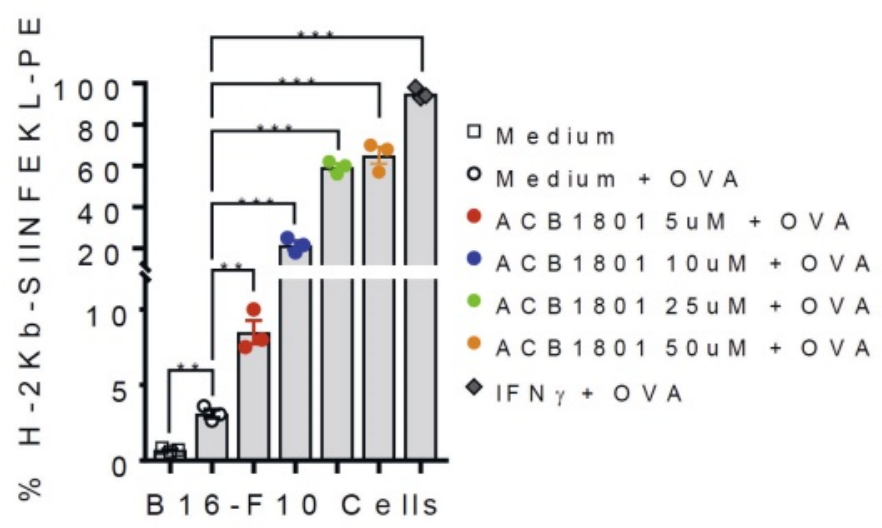

Fig. 4. Effect of ACB1801 on OVA257-264 peptide presentation by MHC-I ( $\mathrm{H}-2 \mathrm{~Kb})$ on the surface of B16-F10 melanoma cells. B16-F10 cells un-pulsed (medium) or pulsed (medium + OVA) with OVA257-264 peptide SIINFEKL for $48 \mathrm{~h}$ were treated simultaneously with ACB-1801 (ACB1801 + OVA) at $5,10,25$ or $50 \mu \mathrm{M}$. Cells pulsed with OVA and treated with IFN-gamma (IFN- $\gamma+$ OVA) were used as a positive control. Cells were then stained with PE anti-mouse H-2Kb bound to SIINFEKL antibody or mouse IgG1, $\kappa$ PE isotype control. Data are reported as the average of 3 independent experiments and shown as mean \pm SEM (error bars). Statistically significant differences (indicated by asterisks) calculated compared to control condition (medium) using an unpaired twotailed Student's t-test are shown $\left({ }^{* *}=p<0.005\right.$ and $\left.^{* * *}=p<0.0005\right)$. 


\subsection{ACB1801 Displays an Immune-dependent Antitumor Activity}

In immune-deficient mice, ACB1801 does not display any antitumor effect at an oral dose of $50 \mathrm{mg} / \mathrm{kg}$. In contrast, a significant antitumor effect is observed in the immunocompetent model (Fig. 5). This is consistent with the fact that the improvement of antigen presentation will trigger the activation of cytotoxic CD8 lymphocytes and subsequent antitumor immune response. This mechanism is evidenced by the modification of the tumor immune landscape with an increase of CD8/T reg ratio upon ACB1801 treatment.
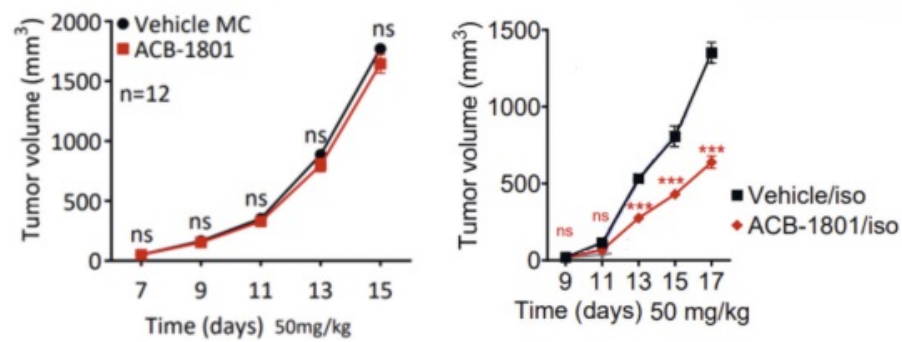

Fig. 5. The antitumor efficacy of ACB1801 in B16-F10 melanoma tumorbearing mice. Left panel: Volume (reported in $\mathrm{mm}^{3}$ ) of B16-F10 melanoma tumors subcutaneously transplanted in immune-deficient NOD Scid Gamma (NSG) mice treated with vehicle (Vehicle MC) or 50 mg/kg ACB1801 (ACB1801) every day per os administration. Right panel: Volume (reported in $\mathrm{mm}^{3}$ ) of B16-F10 melanoma tumors subcutaneously transplanted in immuno-competent C57BL/6 mice treated with vehicle (Vehicle/iso) or 50 mg/kg ACB1801 (ACB-1801/iso) per os administration every day. Treatments were started when tumors became palpable, typically at day 7 or 9 . Left curve represents 3 independent experiments of 4 mice per group. Right curve represents 3 independent experiments of 5 mice per group. All results are shown as mean \pm SEM (error bars). Statistically significant differences (indicated by asterisks) are calculated compared to control conditions using an unpaired two-tailed Student's t-test. Not significant $(n s)=p>0.05$; and ${ }^{\star \star *}=p<0.0005$.

\subsection{Combination of ACB1801 Strongly Potentiates the Anti-PD-1 Efficacy}

When tested alone, anti-PD-1 has no effect on the survival of mice bearing melanoma B16-F10, as expected (Fig. 6). However, in agreement with the results shown in Fig. 4, AB1801 by itself induces a significant but limited increase in mice survival. On the other hand, a striking result is obtained using the combination of ACB1801 and the anti-PD-1 with a strong potentiation of the individual effect.

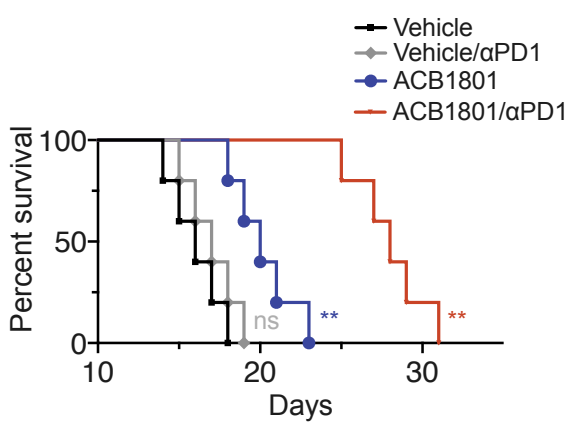

Fig. 6. Combining ACB1801 with anti-PD-1 improves B16-F10 tumor bearing mice survival. Survival of mice treated with vehicle alone (Vehicle), vehicle combined with anti-PD-1 (vehicle/ $\alpha$ PD1), $50 \mathrm{mg} / \mathrm{kg}$ ACB1801 (ACB1801) or anti-PD-1 combined with 50 mg/kg ACB-1801 (ACB1801/ $\alpha$ PD1) was analyzed. Mice survival curves were generated from 5 mice per group. Lack of survival was defined as death or tumor size $>1000 \mathrm{~mm}^{3}$. Mice survival percentage was defined using Graph Pad Prism and $P$ values were calculated using the Log-rank (Mantel-Cox) test (ns $=$ not significant and ${ }^{\star *}=p \leq 0.01$ ).

\section{Conclusion}

In agreement with the clinical observations, we have demonstrated that in an experimental tumor model resistant to checkpoint inhibitors, the improvement of MHC-I-mediated antigen presentation turns a non-responder to a responder phenotype. Considering that in any tumors about $50 \%$ of individual patients display an abnormal antigen presentation, a combination involving a checkpoint inhibitor with a drug as ACB1801 appears to be highly relevant to increase the number of responder patients. ACB1801 works at non-cytotoxic concentration, is active by oral route and extremely well tolerated in animal models and consequently appears to be an attractive drug candidate for a proof of concept clinical trial.

\section{AC BioScience}

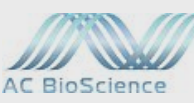

AC BioScience is a startup biotech company with an innovative vision and business model. We are dedicated to developing groundbreaking new therapies to fight a range of cancers and infectious diseases. We have obtained patent protection for one innovative cancer therapy and have filed patents for three others. Two of our four lead molecules in oncology will enter clinical stage in early 2021 . The company has a subsidiary company - AC BioTech SAS - whose offices are located in the Villejuif BioPark near Paris, in proximity to the main French cancer research institutions. AC BioTech serves as a liaison point and vehicle for clinical validation activities in France.

\section{Key Figures}

Founding Year

Location(s)

Legal Form

Founders

Scientific Advisors

No of employees

Market capitalization

\section{Key Inventions}

Beta-carboline derivatives
2017

Headquarter: Ecublens, Switzerland Subsidiary: Villejuif, France

Private limited stock company (Ltd)

Andreas Schläpfer

Prof. Christian Auclair

Prof. Martin Schlumberger

Prof. Patrick Couvreur

Prof. Jean-Pierre Kinet

Prof. Olivier Hermine

Prof. Marco Ciufolini

5

CHF 2.5 million
March 2019: Patent application filed for a Beta-carboline derivative (ACB1801) for the non-toxic and immunological treatment of cancer. 


\begin{tabular}{|c|c|}
\hline S1P lyase inhibitors & $\begin{array}{l}\text { August 2017: Patent application } \\
\text { filed for the enhancement of chemo- } \\
\text { therapy efficiency by Sphingosine- } \\
\text { 1-phosphate (S1P). } \\
\text { August 2019: Patent application filed } \\
\text { for various metabolites of S1P lyase } \\
\text { inhibitors for the treatment of infec- } \\
\text { tious diseases and cancer. } \\
\text { March 2020: Patent application filed } \\
\text { for a combination of flavonoids and } \\
\text { S1P lyase inhibitors for the treatment } \\
\text { of lung inflammation. }\end{array}$ \\
\hline CAP6 & $\begin{array}{l}\text { August 2017: Acquisition of a patent } \\
\text { for a Peptide having the sequence } \\
\text { SEQ.1 for use as a medicament, in } \\
\text { particular for the treatment of cancer. }\end{array}$ \\
\hline RGD-peptide & $\begin{array}{l}\text { March 2020: Patent application filed } \\
\text { for RGD-peptide and use thereof for } \\
\text { inhibiting RGD-binding integrins } \\
\text { and for cancer treatment. }\end{array}$ \\
\hline \multicolumn{2}{|l|}{ Next Milestones } \\
\hline June 2021 & $\begin{array}{l}\text { Start clinical trials with S1P lyase } \\
\text { inhibitors for radiotherapy and/or } \\
\text { chemotherapy }\end{array}$ \\
\hline October 2021 & $\begin{array}{l}\text { Start clinical trials with ACB } 1801 \\
\text { in a combination treatment with anti } \\
\text { PD- } 1 \text { drug }\end{array}$ \\
\hline \multicolumn{2}{|l|}{ Contact } \\
\hline Contact Person & Andreas Schläpfer, CEO \\
\hline Address & $\begin{array}{l}\text { AC BioScience SA } \\
\text { EPFL Innovation Park } \\
\text { Chemin de la Dent d'Oche 1A, } \\
\text { CH-1024, Ecublens }\end{array}$ \\
\hline E-mail & Andreas.schlaepfer@ac-bioscience.com \\
\hline Website & https://www.ac-bioscience.com \\
\hline
\end{tabular}

[1] P. Sharma, S. Hu-Lieskovan, J. A. Wargo, A. Ribas, Cell 2017, 168, 707, https://doi.org/10.1016/j.cell.2017.01.017.

[2] C. Fellner, $P$ T 2012, 37, 503.

[3] D. B. Johnson, C. Peng, J. A. Sosman, Ther. Adv. Med. Oncol. 2015, 7, 97, https://doi.org/10.1177/1758834014567469.

[4] a) D. S. Chen, I. Mellman, Immunity 2013, 39, 1, https://doi.org/10.1016/j. immuni.2013.07.012; b) D. S. Chen, I. Mellman, Nature 2017, 541, 321 , https://doi.org/10.1038/nature21349.

[5] A. M. Goodman, S. Kato, L. Bazhenova, S. P. Patel, G. M. Frampton, V. Miller, P. J. Stephens, G. A. Daniels, R. Kurzrock, Mol. Cancer Ther. 2017, 16, 2598, https://doi.org/10.1158/1535-7163.MCT-17-0386.

[6] R. Abele, R. Tampe, Biochim. Biophys. Acta 1999, 1461, 405, https://doi org/0.1016/s0005-2736(99)00171-6.

[7] A. N. Antoniou, S. J. Powis, T. Elliott, Curr. Opin. Immunol. 2003, 15, 75, https://doi.org/10.1016/s0952-7915(02)00010-9.

[8] E. T. Spiliotis, H. Manley, M. Osorio, M. C. Zuniga, M. Edidin, Immunity 2000, 13, 841, https://doi.org/10.1016/s1074-7613(00)00081-9.

[9] R. Abele, R. Tampe, Physiology (Bethesda) 2004, 19, 216, https://doi. org/10.1152/physiol.00002.2004.

[10] a) F. Garrido, I. Algarra, Adv. Cancer Res. 2001, 83, 117, https://doi org/10.1016/s0065-230x(01)83005-0; b) B. Seliger, T. Cabrera, F. Garrido, S. Ferrone, Semin. Cancer Biol. 2002, 12, 3, https://doi.org/10.1006/ scbi.2001.0404.

[11] L. A. Koopman, W. E. Corver, A. R. van der Slik, M. J. Giphart, G. J. Fleuren, J. Exp. Med. 2000, 191, 961, https://doi.org/10.1084/jem.191.6.961.

[12] A. Ling, A. Lofgren-Burstrom, P. Larsson, X. Li, M. L. Wikberg, A. Oberg, R. Stenling, S. Edin, R. Palmqvist, Oncoimmunology 2017, 6, e1356143, https://doi.org/10.1080/2162402X.2017.1356143.

[13] S. Wang, Z. He, X. Wang, H. Li, X. S. Liu, Elife 2019, 8, https://doi. org/10.7554/eLife.49020.

[14] V. Amsellem, M. H. Kryszke, M. Hervy, F. Subra, R. Athman, H. Leh, C. Brachet-Ducos, C. Auclair, Exp. Cell Res. 2005, 304, 443, https://doi org/10.1016/j.yexcr.2004.10.035.

[15] R. Le Moigne, F. Subra, M. Karam, C. Auclair, Cells 2020, 9, 1168, https:// doi.org/10.3390/cells9051168.

\section{License and Terms}

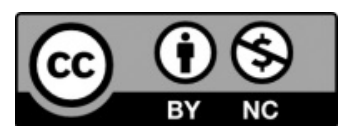

This is an Open Access article under the terms of the Creative Commons Attribution License CC BY_NC 4.0. The material may not be used for commercial purposes.

The license is subject to the CHIMIA terms and conditions: (http:// chimia.ch/component/sppagebuilder/?view=page\&id=12).

The definitive version of this article is the electronic one that can be found at https://doi.org/10.2533/chimia.2020.771 